\title{
Stationary soliton on a charged surface of liquid helium and hydrogen films
}

\author{
A. A. Levchenko, ${ }^{\text {a) }}$ G. V. Kolmakov, L. P. Mezhov-Deglin, and V. B. Shikin \\ Institute of Solid-State Physics, Russian Academy of Sciences, 142432 Chernogolovka, \\ Moscow District, Russia \\ E. Teske and P. Leiderer \\ Universität Konstanz, D78434 Konstanz, Germany
}

(Submitted 6 March 1997)

Pis'ma Zh. Éksp. Teor. Fiz. 65, No. 7, 547-552 (10 April 1997)

A change in the shape of a charged surface of liquid hydrogen and helium - the formation of a solitary wave (a positively charged hump for hydrogen and a negatively charged dimple for helium) - is observed in an electric field exceeding a critical value under conditions of total compensation of the applied field by the surface charge. (C) 1997 American Institute of Physics. [S0021-3640(97)01107-9]

PACS numbers: $67.70 .+\mathrm{n}, 67.55 . \mathrm{Hc}$

It is well known that a flat charged liquid surface in an external perpendicular electric field $E$ becomes unstable when the intensity of the field exceeds a critical value. ${ }^{1}$ Previously the change in the shape of a negatively charged surface of a thick layer of liquid helium (the electrons are localized above the helium) has been investigated in detail in the case when the depth $d$ of the liquid (distance from the free surface to the metal substrate) is much greater than the capillary length $\lambda=\sqrt{\alpha / \rho g}$. Here $\alpha$ and $\rho$ are the surface tension and density of liquid helium and $g$ is the acceleration of gravity. The theoretical calculations have been performed under conditions when the electrons localized above the surface do not completely compensate the applied electric field. It was found that for $d \gg \lambda$ and for a fixed number of charges above the surface, when the field exceeds some critical value a periodic deformation, accompanied by modulation of the surface charge density, with a period of the order of $\lambda$ and a finite amplitude which depends on the degree of supercriticality, ${ }^{2-4}$ arises on the surface of the liquid, i.e., a reconstruction - a transition of the liquid surface from a flat surface into a new stable state - is observed. This phenomenon was studied experimentally in Refs. 5-7.

In the opposite situation, when $d \ll \lambda$, the range of wave numbers at which instability develops starts at $k=0$ (Ref. 8), and in the one-dimensional approximation, as follows from Ref. 9, a localized stationary wave (soliton), whose size depends on the applied field, can arise on the surface.

Until recently it was not clear whether or not it is possible to observe reconstruction of a surface under conditions when the distance between the control electrode and the charged surface of the liquid has a value $d \sim \lambda$, and the electric field is completely compensated by a layer of charge on the surface of the liquid.

The surface of liquid helium and hydrogen can be charged, for example, by local- 
izing electrons above the surface or positive ions beneath the surface. The problems of the stability and reconstruction of negatively or positively charged liquid surfaces are equivalent. For this reason, in this letter experiments with a negatively charged helium surface are combined with experiments with a positively charged hydrogen surface.

This letter reports the experimental observation of a stationary reconstructed surface of charged helium and hydrogen films under conditions when the charges completely compensate the applied electric field and presents data on the shape of the reconstructed surface. The transition from a flat state into a new reconstructed state is "soft." It is found that the solitary stationary axisymmetric wave (soliton) corresponding to a dimple on a negatively charged liquid-helium surface (electrons above the helium) and a hump on a positively charged liquid-hydrogen surface (positive charges localized beneath the surface) is a stable form of the reconstructed surface.

\section{EXPERIMENTAL PROCEDURE}

The properties of a negatively charged liquid-helium surface in the normal state and a positively charged liquid-hydrogen surface were studied in the experiments.

The experimental cells had the form of flat capacitors (diodes) arranged horizontally in cylindrical optical containers. The distance between the capacitor plates was equal to $8 \mathrm{~mm}$ in the experiments with liquid helium and $6 \mathrm{~mm}$ in the experiments with liquid hydrogen. The diameter of the plates $(D \geqslant 20 \mathrm{~mm})$ was several times greater than the distance between the plates. The liquid was condensed on the bottom plate of the capacitor. The liquid column was 0.1 and $0.15 \mathrm{~mm}$ high for helium and $3 \pm 0.1 \mathrm{~mm}$ high for hydrogen. We recall that the capillary length in helium $\lambda=0.4 \mathrm{~mm}$ at $T=4.2 \mathrm{~K}$ and $\lambda=1.8 \mathrm{~mm}$ at $17 \mathrm{~K}$ for hydrogen, i.e., the distance $d$ between the control electrode (top plate in hydrogen, bottom plate in helium) and the free surface of the liquid was comparable to the capillary length $\lambda$.

The liquid-helium surface was charged with electrons with the aid of a pointed tip placed in vapor in the presence of a perpendicular compressing field. The change in the shape of the surface was followed according to the change in the interference pattern produced by two laser beams reflected from the bottom half-transmitting (metallized) plate of the diode and the top, mirror plate. The observational procedure is described in Ref. 10, for example.

In the experiments with liquid hydrogen the bottom plate, coated with a layer of radioactive material, of the diode served as the source of charge. Under the action of the applied electric field the positive charges $\left(\mathrm{H}_{2}^{+}\right.$ions surrounded by a layer of solidified hydrogen) was pressed against the surface located at a distance $d$ from the top metal electrode; in this case, virtually no current flowed through the surface. The experimental arrangement is described in Ref. 11. The shape of the charged surface was monitored with a TV camera, which gave an image of the profile of the liquid in the gap between the plates of the diode. A change in the shape of the surface with increasing electric field was also judged according to the deflection of the laser beam reflected from the surface of the liquid.

We underscore the fact that the measurements were performed under conditions of a bounded geometry (cylindrical ampul with a diameter several times greater than the 
interelectrode distance) and the total amount of liquid in the helium and hydrogen films in the experimental cell remained unchanged.

\section{EXPERIMENTAL RESULTS}

For low voltages between the diode plates the equipotentially charged surface of the liquid curves smoothly. When a critical voltage $U_{c 1}$ is reached, a macroscopic section (a dimple on the surface of helium and a hump on the surface of hydrogen) with a characteristic diameter of several millimeters appears on the surface. It should be noted that in both helium and hydrogen the reconstructed section could arise on the surface at random points. In the experiments with hydrogen an a single hump could be moved to the center of the diode by slightly changing the inclination of the cell.

Figure 1 shows two interference patterns of the charged surface of a liquid-helium film - before and after the appearance of a deformed section (marked by arrows in Fig. 1b). The diameter of the dark spot at the center of the deformed section equals $1.5 \mathrm{~mm}$. The potential difference between the diode plates was $U=170 \mathrm{~V}$, the measurement temperature $T=4.2 \mathrm{~K}$, and the liquid layer was $0.15 \mathrm{~mm}$ thick. The charge density far from the deformed section remained practically unchanged. This follows from the fact that the distances between the interference fringes at the edges of the charged surface are the same. This is specially noted in order to underscore the fact that the liquid surface remains an equipotential surface after it is deformed.

The deformed section was stable for $1.2 \mathrm{~s}$, after which it discharged on the bottom plate of the diode. For a $0.1 \mathrm{~mm}$ thick liquid layer and $U=400 \mathrm{~V}$ the lifetime of the deformed section decreased to $0.4 \mathrm{~s}$, but once again the lifetime was much longer than the characteristic relaxation time of disturbances in the electron layer above the helium surface.

Figure 2a shows a photograph of the equilibrium profile of a reconstructed surface of a layer of liquid hydrogen with the potential difference between the plates of the diode $U=1620 \mathrm{~V}$ and temperature $T=17 \mathrm{~K}$. The points in Fig. $2 \mathrm{~b}$ were obtained by scanning this profile. In a constant field the form of the profile remained unchanged over an observation time of longer than $10^{3} \mathrm{~s}$.

The height of the deformed section of the surface (hump) at the center of the diode could be regulated by varying the voltage between the diode plates in the range $U=1300-1700 \mathrm{~V}$. No surface reconstruction was observed for subthreshold voltages $U\left(U_{c 1} \approx 1300 \mathrm{~V}\right.$ at the given temperature). At voltages above $1750 \mathrm{~V}$ the reconstructed surface becomes unstable and a discharge is observed (second critical voltage $U_{c 2}$ ). At the moment of the discharge, of the order of $10^{8}$ charges arrive at the collector. After the discharge the surface relaxes to the initial flat state. Then the process repeats. In Ref. 11 we reported the observation of quasiperiodic oscillations of the charge surface of liquid hydrogen in strong fields. A similar phenomenon - the appearance of ionic jets (geysers) on the surface of superfluid helium in strong fields - was observed in Ref. 12.

Figure 3 displays the maximum angle of reflection $\beta$ of a laser beam from the liquid surface, measured at a temperature of $14.6 \mathrm{~K}$, versus the voltage $U$. One can see that the dependence of the reflection angle on the control voltage $\beta(U)$ changes substantially at voltages above the first critical voltage (in the present measurements $U_{c 1} \approx 1500 \mathrm{~V}$ ). 


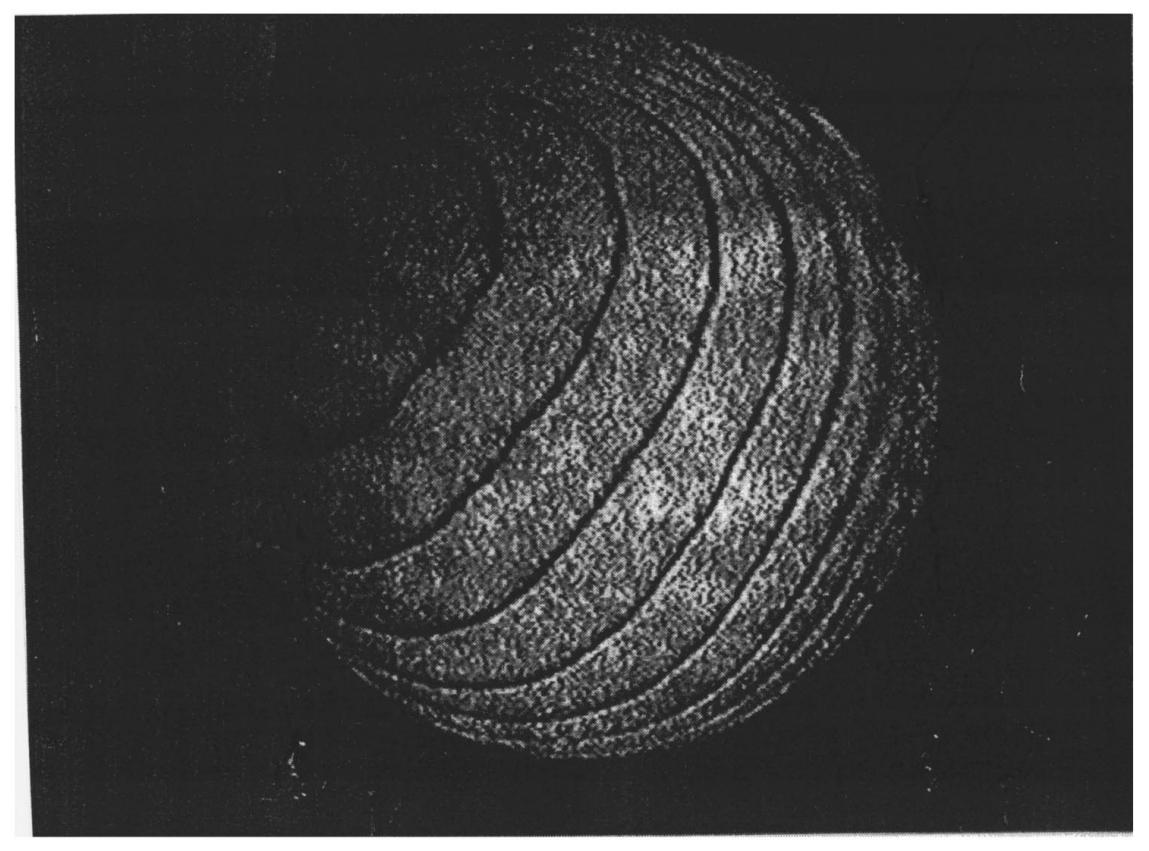

a

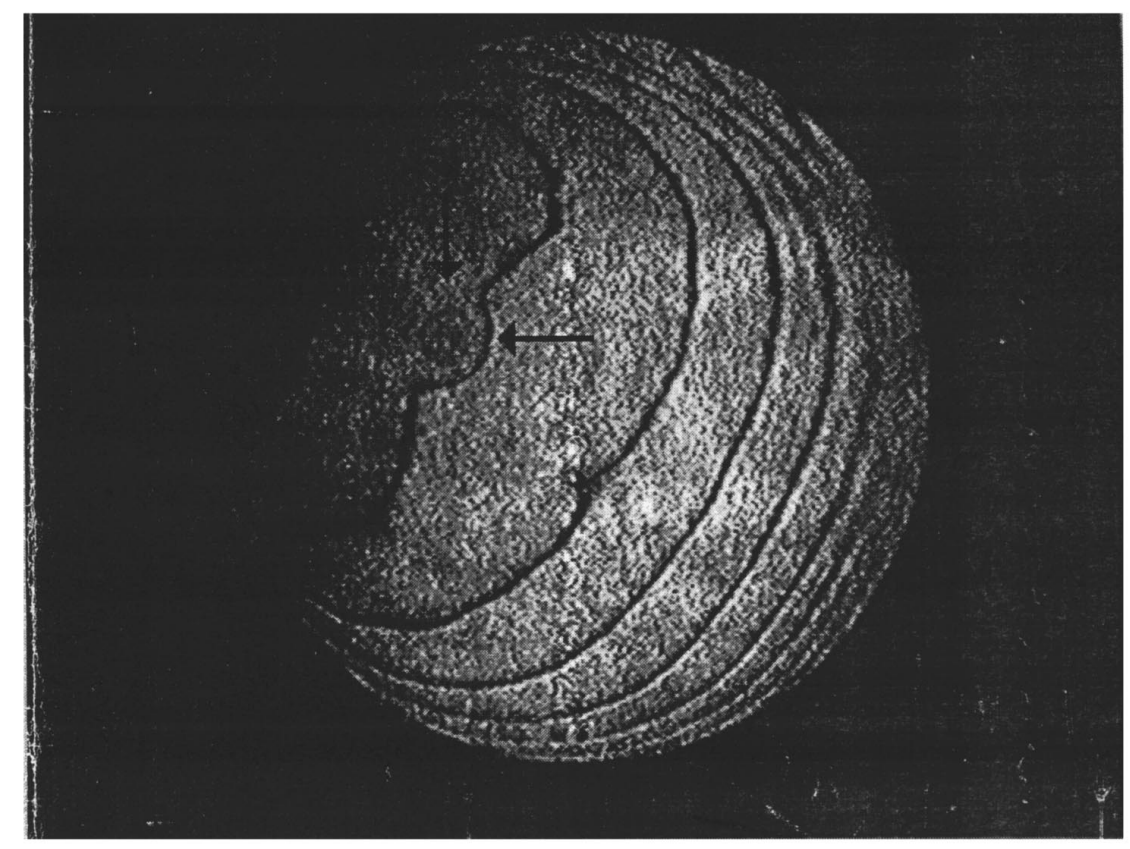

b

FIG. 1. Interferogram of an exponentially charged liquid-helium surface before (a) and after (b) the formation of a dimple (marked by arrows). Temperature of the liquid $-4.2 \mathrm{~K}$. Diameter of the visible section of the liquid $-20 \mathrm{~mm}$. 

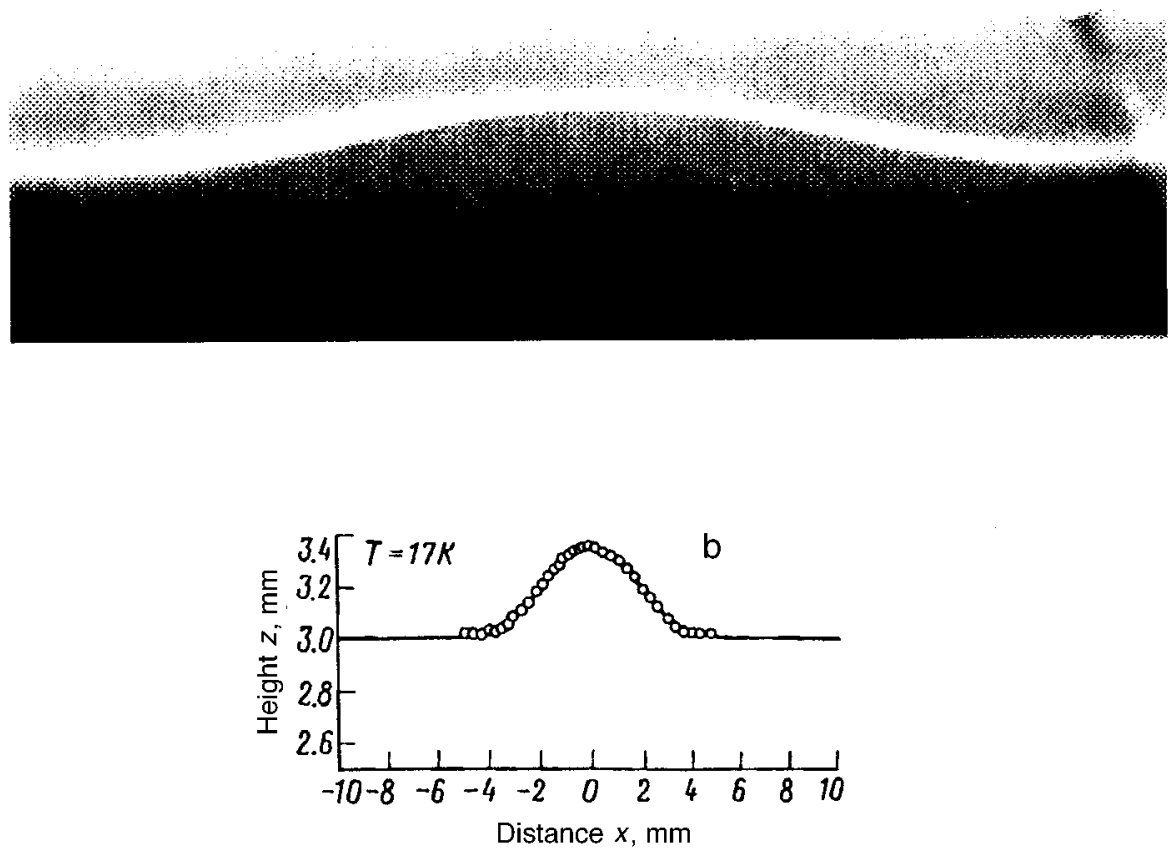

FIG. 2. a) Photograph of the profile of a reconstructed equipotentially charged surface of liquid hydrogen. The linear size in the horizontal direction equals $10 \mathrm{~mm}$. Temperature of the liquid $-17 \mathrm{~K}$. b) Profile obtained for the reconstructed section (hump) by scanning the photograph (circles). Solid line - the function (1) with the parameters $A=0.38 \mathrm{~mm}$ and $R=2.5 \mathrm{~mm}$.

\section{DISCUSSION OF THE RESULTS}

First the following important question must be answered. Do the observations repeat the experiments of Ref. 10 where dimples were observed on the surface of a thick $(d \gg \lambda)$ helium film on a metal substrate? The answer is unequivocally no.

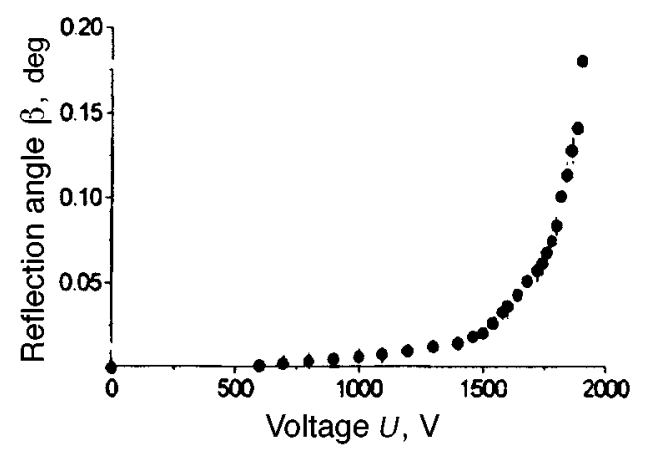

FIG. 3. Maximum angle of reflection of a laser beam from a charged liquid-hydrogen surface as a function of the voltage $U$. The temperature of the liquid is equal to $14.6 \mathrm{~K}$. 
The experimental situation in our work is qualitatively different from the situation observed in Ref. 10 and discussed in Refs. 3 and 4. In our experiments the charge density is determined by the applied voltage (the charges completely compensate the external electric field) and equals the maximum possible density $n=n_{c}=E^{2} / 4 \pi$ on the entire surface, while in Ref. 10 the charge density on a flat surface far from the reconstructed section (dimple) is close to zero, $n \ll n_{c}$. Moreover, in the experiments with hydrogen the number of charges beneath the surface varies and increases with the applied voltage.

The observations showed that the lifetime of the reconstructed helium and hydrogen surfaces is quite long. This makes it possible to talk about the observation of a stationary charged solitary wave (a dimple in helium or a hump in hydrogen).

A number of qualitative conclusions can be drawn from the experimental results (Figs. 1 and 2).

1) Surface reconstruction in a stationary electric field in a finite-diameter cell with $d \sim \lambda$ is possible with both fixed (helium) and variable (hydrogen) numbers of charges at the surface.

2) Under conditions when the charge density on an equipotential surface is fixed by an external field, a solitary stationary wave - a dimple in the experiments with electrons above a helium surface or a hump in experiments with positive charges beneath a hydrogen surface - arises on the surface in fields exceeding a critical value $U_{c 1}$. The possibility of the appearance of such a wave is predicted in a one-dimensional approximation in Ref. 9.

3) As the field increases further, a pulsed discharge is observed, i.e., there exists a second critical field (and, correspondingly, a voltage $U_{c 2}$ ) above which the reconstructed surface of the liquid is unstable.

In Ref. 9 the one-dimensional case corresponding to the formation of a periodic system of "rolls" on an infinite surface of a thin helium film $(d \ll \lambda)$ in a field exceeding some critical field is studied. The shape of a solitary roll (1D soliton), corresponding to an infinitely long period (for the experiment, much greater than the diameter of the experimental cell), is described by the expression

$$
z(x)=A \cosh ^{-2}(x / R),
$$

where $A$ is the amplitude and $R$ is the characteristic size of the soliton.

Unfortunately, the computational results obtained in Ref. 9 cannot be used to describe a real, substantially non-one-dimensional situation. Nonetheless, it was found that the form of the hump observed in experiments with liquid hydrogen is described satisfactorily by expression (1), provided that the hump is axisymmetric and $x$ is replaced by the distance $r$ from the center of the soliton. Then $R$ is the characteristic radius of the soliton. Numerical fitting of the profile of the hump (points in Fig. 2b) gave the values $A=0.38 \mathrm{~mm}$ and $R=2.5 \mathrm{~mm}$ (solid curve in Fig. 2b).

The evolution of the shape of the hump with increasing applied voltage can also be judged from the curve $\beta(U)$ in the experiment with hydrogen. From the plot shown in Fig. 3 it is evident that there exist two different regimes of variation of the shape of the free surface of the liquid - below and above the first critical field $U_{c 1}$. The initial section 
$\left(U<U_{c 1}\right)$ corresponds to the standard drawing of the charged liquid into the field of the capacitor, the reflection angle being proportional to the square of the applied voltage: $\beta \propto U^{2}$. In fields above the first critical field, i.e., in the region $U_{c 1}<U<U_{c 2}$, the experimental dependence can be described by the relation $\beta \approx\left(U-U_{c 1}\right)^{m}$, where the exponent $2<m<3$. This section of the curve $\beta(U)$ describes the change in the slope of the lateral surface of the soliton with increasing voltage $U$.

In summary, the reconstruction of an equipotential charged surface of a liquid in a stationary electric field above a critical field was observed in the experiments under the conditions $d \sim \lambda$, where $d$ is the distance between the charged surface and the control electrode and $\lambda$ is the capillary length. For further analysis of the observed phenomenon it could be important that the measurements were performed in a finite-size flat capacitor with diameter much greater than the characteristic lengths $d$ and $\lambda$.

This work was supported in part by grants from NASA-RKA (Project No. TM-17) and INTAS-93 (Project No. 93-933).

a)e-mail: levch@issp.ac.ru

${ }^{1}$ L. D. Landau and E. M. Lifshitz, Electrodynamics of Continuous Media, Pergamon Press, New York [Russian original, Nauka, Moscow, 1982].

${ }^{2}$ L. P. Gor'kov and D. M. Chernikova, JETP Lett. 18, 68 (1973); Dokl. Akad. Nauk SSSR 228, 829 (1976) [Sov. Phys. Dokl. 21, 328 (1976)].

${ }^{3}$ V. I. Mel'nikov and S. V. Meshkov, Zh. Éksp. Teor. Fiz. 82, 1910 (1982) [Sov. Phys. JETP 55, 1099 (1982)].

${ }^{4}$ V. B. Shikin and Yu. P. Monarkha, Two-Dimensional Charged Systems in Helium [in Russian], Nauka, Moscow, 1989.

${ }^{5}$ P. Leiderer, Phys. Rev. B 20, 4511 (1979).

${ }^{6}$ M. Wanner and P. Leiderer, Phys. Rev. Lett. 42, 315 (1979).

${ }^{7}$ P. Leiderer, M. Wanner, Phys. Rev. Lett. A 73, 185 (1979).

${ }^{8}$ D. M. Chernikova, Fiz. Nizk. Temp. 2, 1374 (1976) [Sov. J. Low Temp. Phys. 2, 669 (1976)].

${ }^{9}$ V. B. Shikin and P. Leiderer, Fiz. Nizk. Temp. (in press).

${ }^{10}$ P. Leiderer, W. Ebner, and V. B. Shikin, Surf. Sci. 113, 405 (1982).

${ }^{11}$ A. A. Levchenko and L. P. Mezhov-Deglin, Fiz. Nizk. Temp. 22, 46 (1996) [Low Temp. Phys. 22, 33 (1996)].

${ }^{12}$ V. P. Volodin and M. S. Khaı̆kin, JETP Lett. 30, 572 (1979).

Translated by M. E. Alferieff 